\title{
MEJORA DE SISTEMA DE CONTROL COORDINADO DE ROBOTS MÓVILES MEDIANTE LA UTILIZACIÓN DE UN SISTEMA DE LOCALIZACIÓN POR VISIÓN
}

\author{
Pablo Álvarez Benito, David Gallarta Sáenz, Javier Rico-Azagra, Montserrat Gil-Martínez \\ \{pablo.alvarez, david.gallarta\}@alum.unirioja.es, \{javier.rico,montse.gil\}@unirioja.es \\ Grupo de Investigación de Ingeniería de Control, Departamento de Ingeniería Eléctrica, \\ Universidad de La Rioja
}

\begin{abstract}
Resumen
El trabajo presenta un sistema de control coordinado de cobertura utilizando el algoritmo de Lloyd en el que intervienen tres robots LEGO Mindstorms EV3, como ejemplo práctico ilustrativo de robótica educativa. La aplicación se desarrolla en MATLABSimulink, un entorno conocido por los estudiantes, al utilizarse habitualmente para el control de sistemas dinámicos. Se pretenden mostrar los problemas surgidos por la utilización de sistemas de localización poco precisos, en este caso, los encoders incorporados en los motores de los robots. Para solucionar este problema se utiliza un sistema de localización mediante la captura de datos por visión. Se explica la infraestructura hardware y software empleada en el laboratorio que alberga el sistema de visión. Este conjunto ofrece una plataforma de bajo coste para sistemas de localización.
\end{abstract}

Palabras clave: Control coordinado, Control de cobertura, Robots móviles, Sistema de localización por visión.

\section{INTRODUCCIÓN}

La robótica educativa ha experimentado un notable crecimiento en los últimos años, en un proceso en paralelo a la llegada de los robots a nuestro día a día. El interés que despierta un robot en niños y jóvenes facilita la primera toma de contacto, lo cual se fomenta desde instituciones con cursos o competiciones de robots. Otro apoyo importante surge gracias a la aparición de kits de robots que no requieren conocimientos en electrónica o programación avanzada, por lo que pueden utilizarse en diversos rangos de edad. Un ejemplo es el LEGO Mindstorms, sobre el cual más adelante se hablará en profundidad.

Uno de los objetivos de la robótica educativa [19] es cambiar la percepción negativa que parte de los alumnos tienen de las asignaturas de ciencias técnicas, haciéndolas más dinámicas y atractivas. Sin embargo, la robótica como herramienta para la educación busca transmitir conocimientos más allá de las habilidades tecnológicas, trabajando competencias necesarias en la sociedad actual. Algunas de ellas son:

- Potenciar la creatividad en el planteamiento de soluciones frente al problema propuesto, como origen de la innovación.

- Desarrollar el pensamiento lógico, a través de la concordancia entre lo programado y las tareas que se quieren ejecutar.

- Emplear el error como herramienta de aprendizaje, lo cual posibilita la mejora de soluciones y la superación personal.

- Trabajar en equipo para estimular las habilidades sociales.

En el ámbito académico, las prácticas de laboratorio suponen un elemento fundamental para la adquisición de las competencias necesarias. Gracias a los kits mencionados anteriormente, estas prácticas significan un contacto real para el alumno con lo que se van a encontrar más allá de las aulas, lo cual supone una ventaja frente a los entornos de simulación. La mayoría de estos robots educativos se engloban dentro de los robots móviles, sobre los cuales pueden plantearse retos tecnológicos similares a los que se va a enfrentar la industria en los próximos años, tales como los problemas de estimación de actitud [18] y posición [14], la coordinación de vehículos o la comunicación entre flotas.

Centrándose en el tema sobre el que trata este trabajo, los sistemas multi-robot $[1,3,5,7]$ abren un nuevo campo de estudio en la robótica móvil, ya que permiten la optimización de multitud de aplicaciones, facilitando la consecución de objetivos difícilmente alcanzables para un único robot. Entre sus ventajas destacan su versatilidad y su flexibilidad en las tareas que pueden realizar, permitiendo el trabajo en áreas más grandes y alcanzado los objetivos planteados de una manera más eficiente.

Otra característica importante que tienen estos sistemas es la redundancia: si se produce un fallo en uno de los agentes, todavía queda un número determinado de unidades que pueden continuar realizando las tareas programadas. Existen 
principalmente dos tipos de control coordinado: el control de formación [15] y el control de cobertura [17]. En el primer caso, el objetivo es hacer que los robots se posicionen de una determinada forma con respecto a los demás para realizar un movimiento coordinado manteniendo esa formación. En el segundo, se trata de cubrir un área determinada, repartiendo el espacio entre varios robots, lo que reduce el tiempo necesario para alcanzar los objetivos deseados.

El presente trabajo se organiza en cinco secciones. En la segunda se describe la plataforma del robot empleado, sus elementos y modo de empleo. La tercera sección presenta el sistema de control desarrollado en Simulink y los resultados obtenidos. La cuarta enseña el sistema de posicionamiento por visión, que mejora el sistema de control implementado y los resultados obtenidos. Por último, la quinta contiene las conclusiones.

\section{LEGO MINDSTORMS EV3}

La plataforma empleada para el desarrollo del sistema de control es LEGO Mindstorms EV3, un kit enfocado a la educación, empleado tradicionalmente en cursos de educación secundaria para un acercamiento la robótica [13]. Su bajo coste lo hace muy accesible cuando el presupuesto disponible no permite obtener, por su elevado precio, otro tipo de robots útiles para la docencia. No obstante, la existencia de una librería de bloques específicos para esta familia de robots en Simulink, posibilita que puedan experimentarse con ellos técnicas empleadas en robots industriales, por lo que este robot constituye una herramienta de trabajo excepcional para alumnos a nivel universitario. Además, MATLAB-Simulink [4] es un entorno de programación con el cual los alumnos se encuentran familiarizados por ser una herramienta de trabajo habitual en varias asignaturas.

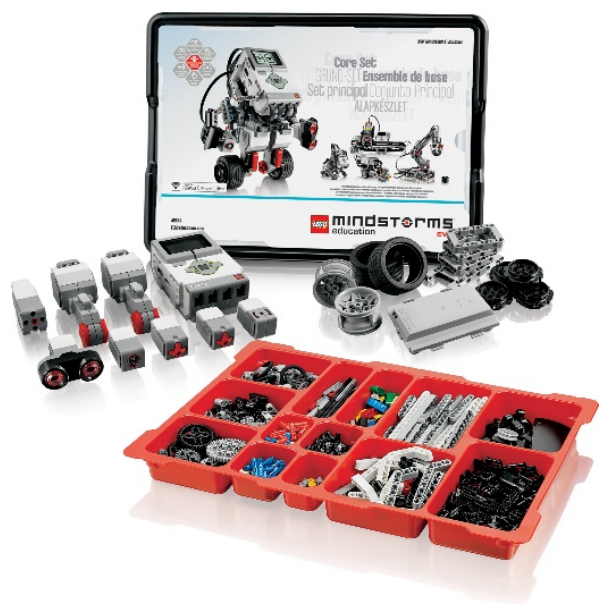

Figura 1: Kit LEGO Mindstorms EV3
La plataforma LEGO Mindstorms EV3 presenta una serie de elementos electromecánicos que se controlan desde el ordenador (mediante USB, Wi-Fi o Bluetooth), los cuales se pueden montar con múltiples piezas y accesorios. Al desarrollarse con un fin educativo, no requiere conocimientos técnicos relacionados con la electricidad o la electrónica, por lo que permite centrarse en el aprendizaje y los experimentos robóticos a pequeña escala. A través de los múltiples accesorios (sensor de color, sensor táctil, sensor ultrasónico...) que incorpora, es posible conocer técnicas empleadas a mayor escala en la industria. Por este motivo, como su montaje se realiza desde cero con las diferentes piezas disponibles, permite obtener diferentes configuraciones según el uso que se le quiera dar. Esta versatilidad implica también que no existan muchas dificultades a la hora de reparar el robot, ya que basta con sustituir la pieza dañada. Esto, sumado a la facilidad para encontrar repuestos, hace que su mantenimiento sea sencillo.

A pesar de las ventajas comentadas anteriormente, la plataforma LEGO Mindstorms EV3 no se encuentra carente de defectos. Sus principales problemas son la escasa potencia de sus motores y la baja precisión de los sensores incorporados, que dan lugar a importantes errores cuando se estima el estado del vehículo empleando únicamente técnicas basadas en odometría. En este caso, la utilización del sistema de captura de datos por visión, del que se habla posteriormente, soluciona dicho problema.

\section{CONTROL DE COBERTURA BASADO EN ENCODERS}

En esta sección se presenta una solución al problema de control de cobertura de un área con tres robots LEGO Mindstorms EV3.

\subsection{ROBOT DIFERENCIAL Y SU CONTROL COMO UNIDAD ELEMENTAL}

En este caso se configuran los robots EV3 para que trabajen en modo diferencial [8], es decir, empleando dos ruedas situadas sobre un eje común, acopladas cada una a su propio motor, que permite controlarlas de forma independiente. Este tipo de robot es el más utilizado en laboratorios docentes [6] por la simplicidad de su diseño, su tamaño reducido, su capacidad de maniobra y su asequible precio. A pesar de su sencillez existen retos importantes en su control. Permite desarrollar numerosas competencias educativas, lo que hace de este robot una gran herramienta para el aprendizaje de los alumnos.

Estos robots pueden desplazarse en línea recta, describiendo un arco o girando sobre su propio eje. Los giros se realizan cambiando la velocidad relativa 
de cada rueda. Para proporcionar estabilidad incorporan una rueda esférica de apoyo.

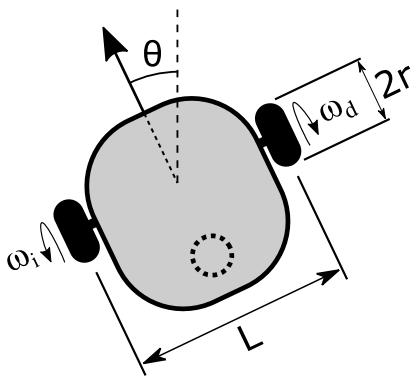

Figura 2: Esquema del robot diferencial

El movimiento del robot se estudia respecto al punto central de su eje. Las variables de entrada requeridas para su control son la velocidad angular de las ruedas $\left(\omega_{d}\right.$ y $\left.\omega_{i}\right)$, que combinadas permiten controlar la velocidad lineal

$$
v=\frac{r}{2} \cdot\left(\omega_{d}+\omega_{i}\right)
$$

y angular del robot

$$
\omega=\frac{r}{L} \cdot\left(\omega_{d}+\omega_{i}\right)
$$

siendo $L$ la distancia entre los centros de las ruedas y $r$ su radio. Resolviendo el sistema formado por las ecuaciones (1) y (2) se obtienen las velocidades angulares de cada rueda en función de la velocidad lineal y la velocidad angular del robot.

Para realizar el control del robot diferencial, es necesario implementar una estructura de control jerárquica con varios niveles. En el primer nivel se sitúan los lazos de control de velocidad de cada una de las ruedas. Para ello, el alumno debe realizar la identificación experimental del modelo salida-entrada y diseñar un controlador apropiado. El esquema de control se muestra en la Figura 3.

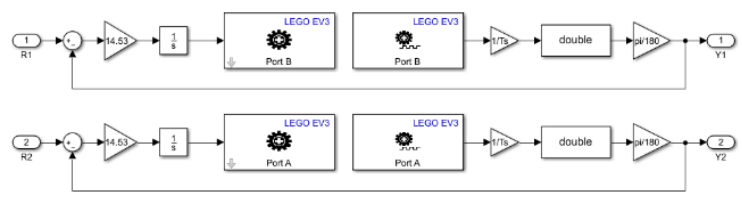

Figura 3: Esquema de control de los motores de cada robot con bloques Simulink

En un segundo nivel jerárquico se encuentran los lazos de control encargados del movimiento del robot. Estos calculan la velocidad lineal $(v)$ y angular $(\omega)$ aplicada al robot para que este alcance la posición $(x, y)$ deseada. Para lograrlo se combinan dos lazos de control: uno para la dinámica longitudinal (control de v) y otro para la dinámica lateral (control de $\omega$ ), los cuales emplean ganancias de control proporcionales, $K_{v}$ y $K_{\omega}$, respectivamente. Para cerrar dichos lazos de control pueden emplearse técnicas basadas en odometría a partir de los encoders o la información generada por las cámaras del sistema OptiTrack, explicado posteriormente. La Figura 4 muestra el modelo Simulink empleado en la aplicación propuesta y ejecutado en los tres robots utilizados.

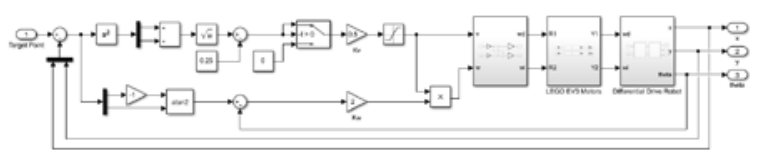

Figura 4: Esquema de control del movimiento de cada robot con bloques Simulink

\subsection{ALGORITMO DE CONTROL DE COBERTURA}

El control de cobertura implementado busca que los tres robots empleados en el experimento cubran un área determinada de una manera eficiente. Para ello, se divide esta zona en tantas porciones como número de robots se utilicen, intentando que la extensión de los espacios asignados a cada robot sea similar.

La técnica empleada se basa en el algoritmo de Lloyd [7], un algoritmo iterativo que busca encontrar una teselación de Voronoi centroidal [9] para un conjunto de puntos dentro de un área determinada. Una teselación de Voronoi centroidal es un tipo especial de diagrama de Voronoi en el que el punto que genera cada una de las celdas coindice con el centroide de la misma, obteniendo así una distribución uniforme de las celdas. En cada iteración, el algoritmo de Lloyd genera el diagrama de Voronoi del conjunto de puntos dado, calcula el centroide de cada una de las celdas resultantes y mueve cada punto hacia el centroide de su celda correspondiente para generar un nuevo diagrama de Voronoi. El algoritmo se repite hasta la obtención de una teselación de Voronoi centroidal.

Este algoritmo presenta una importante característica que hace muy sencilla su implementación: no existe la posibilidad de que se produzcan colisiones entre los robots. Esto se debe a que los puntos de referencia hacia los que se tiene que desplazar cada robot siempre se encuentran dentro de su propia celda, por lo que las trayectorias de los robots no se cruzan en ningún momento. Se ha implementado en Simulink, tomando como puntos de entrada las posiciones en el plano de cada uno de los robots. Las salidas del mismo son las coordenadas a las que debe desplazarse cada robot.

El algoritmo se ejecuta de manera centralizada en un PC encargado de procesar y coordinar la información. Por ello, se deben desarrollar $n+1$ modelos Simulink, 
uno para cada uno de los $n$ robots que trabajan en la aplicación y otro para el PC. El modelo Simulink que se ejecuta en el PC puede emplearse en tareas de monitorización o para el desarrollo de las estrategias de control.

\subsection{RESULTADOS PRELIMINARES}

A continuación, se ilustran los resultados obtenidos en un primer experimento. El lazo de control interno de cada uno de los robots encargado del movimiento del robot se cierra empleando la información generada por sus encoders, con el fin de ilustrar los problemas de este sistema de posicionamiento relativo. Como puede apreciarse en la Figura 5, cada uno de los robots se ha desplazado en busca de sus sucesivos puntos de referencia (color negro), asignados por el algoritmo de control de cobertura.

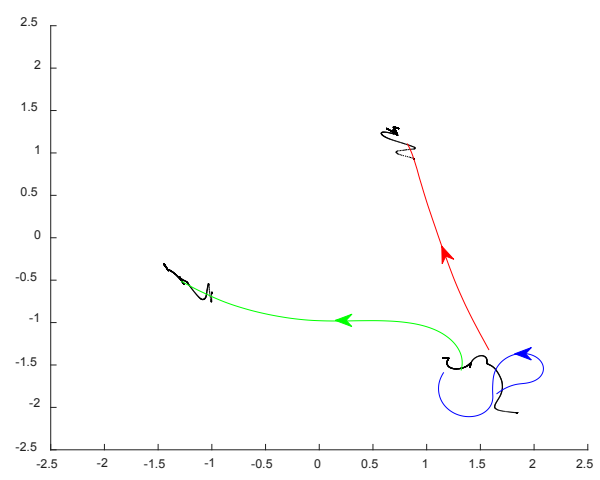

Figura 5: Trayectoria descrita por cada robot hasta alcanzar su posición final

Si no se realiza un análisis exhaustivo de los resultados, puede parecer que los robots han logrado su objetivo. Sin embargo, la distorsión producida por una estimación incorrecta de la posición de los robots debida a la acumulación de errores de los encoders produce que la cobertura resultante no sea adecuada. Como puede comprobarse en la Figura 6 derecha, las celdas ocupadas por cada uno de los robots presentan diferente tamaño. El ejemplo más claro se da en la celda ocupada por el robot de la esquina inferior derecha (color rosa), que es notablemente más pequeña que resto.
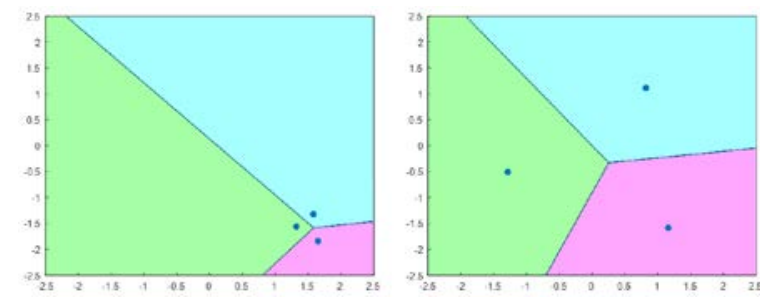

Figura 6: Distribución inicial y final de las celdas para cada robot

\section{CONTROL DE COBERTURA BASADO EN SISTEMA DE VISIÓN}

Para solventar los problemas anteriores derivados de una estimación de la posición poco precisa, la cual acumula errores, en un segundo experimento se utiliza un sistema de visión instalado en un laboratorio para la obtención de la posición absoluta de los robots. Este sistema consta de tres elementos: el área de trabajo, el sistema de adquisición de datos por visión $[2,12]$ y la arquitectura software que soporta el sistema. Estos elementos se describen a continuación.

\section{1 ÁREA DE TRABAJO Y SISTEMA DE ADQUISICIÓN DE DATOS POR VISIÓN}

El área de trabajo habilitada tiene una extensión aproximada de $7 \mathrm{~m} \times 7 \mathrm{~m}$, espacio suficiente para efectuar ensayos tanto con robots móviles terrestres [16] como con pequeños UAVs (Unmanned Aerial Vehicle) de tipo multi-rotor. En el perímetro de este recinto se ubican 8 cámaras Flex 3 (ver Figura 7), comercializadas por OptiTrack [10]. Su ubicación y orientación son estratégicas para cubrir la mayor área posible, teniendo en cuenta que un punto debe ser visto por al menos tres cámaras.

Este laboratorio, junto con los robots LEGO EV3, puede utilizarse a partir de tercer curso de grado, una vez que los conocimientos sobre MATLAB-Simulink $\mathrm{y}$ estrategias de control son suficientemente avanzados. Hasta el momento, se ha empleado en una asignatura optativa de cuarto curso, así como en trabajos fin de grado.

También debe comentarse que en el suelo del área de trabajo se ha instalado una moqueta de color gris (ver Figura 7 derecha) para reducir la reflectancia del suelo, evitar el deslizamiento de las ruedas de los robots móviles y amortiguar el impacto en el caso de que un UAV se estrelle contra el suelo.
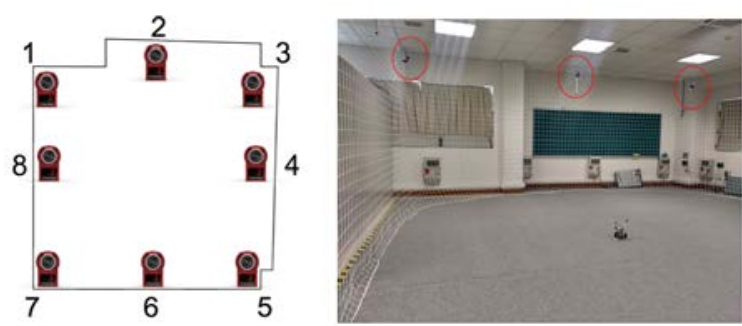

Figura 7: Disposición de las cámaras en el laboratorio

Una vez calibrado, el sistema trabaja con errores de estimación de posición y de orientación inferiores a $0,2 \mathrm{~mm}$ y $0,1^{\circ}$. Siguiendo esta configuración, se obtiene un área de captura de $5 \mathrm{~m} \times 5 \mathrm{~m}$. 
Para que los objetos puedan ser reconocidos por las cámaras, deben tener distribuidos, de manera aleatoria por su estructura, marcadores reflectantes proporcionados por OptiTrack. La Figura 8 muestra la colocación de los marcadores en un LEGO Mindstorms EV3 y su representación en Motive, software comercializado por OptiTrack, como un cuerpo rígido.
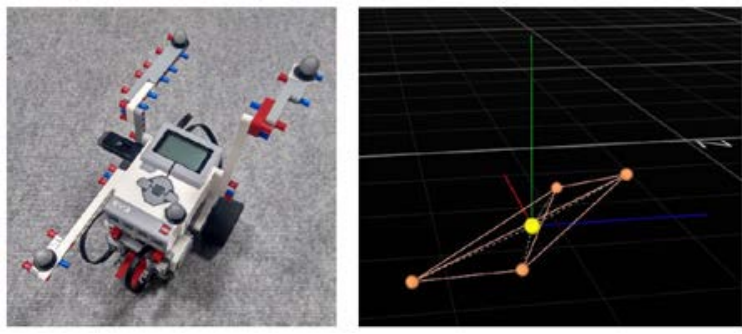

Figura 8: Marcadores en un robot LEGO EV3 y representación en Motive

El software Motive es una plataforma cerrada que se utiliza para procesar la información obtenida por las cámaras en tiempo real. Esta información corresponde a los seis grados de libertad, tres para la posición y tres para la orientación, que definen el estado de un cuerpo rígido, los cuales son obtenidos a partir de sus marcadores. La información se puede transmitir a Simulink mediante una S-Function que se puede programar para obtener los datos deseados.

\subsection{ARQUITECTURA SOFTWARE Y SISTEMA DE COMUNICACIONES}

La información obtenida por las cámaras llega al PC empleando dos concentradores OptiHub, a cada uno de los cuales se conectan cuatro cámaras mediante USB. A su vez, estos se conectan entre sí para la sincronización de los datos capturados. El software Motive gestiona automáticamente la información y estima la posición de los cuerpos. Los datos generados por Motive son retrasmitidos internamente empleando una VLAN (Virtual Local Area Network) de tipo C generada con un router. A esta red se conectan mediante enlaces Wi-Fi los robots LEGO EV3 y el PC en el que se ejecutan las pruebas. Se debe tener en cuenta que la transmisión de paquetes entre el PC y los robots EV3 se realiza empleando el protocolo UDP.

Puede comprobarse cómo con esta arquitectura se centraliza en tiempo real en el PC toda la información generada. De este modo, el PC actúa como emisor y receptor enviando la información necesaria a cada elemento del sistema. El sistema de comunicaciones empleado para enviar y recibir información entre los diferentes elementos que componen el sistema se muestra en la Figura 9.

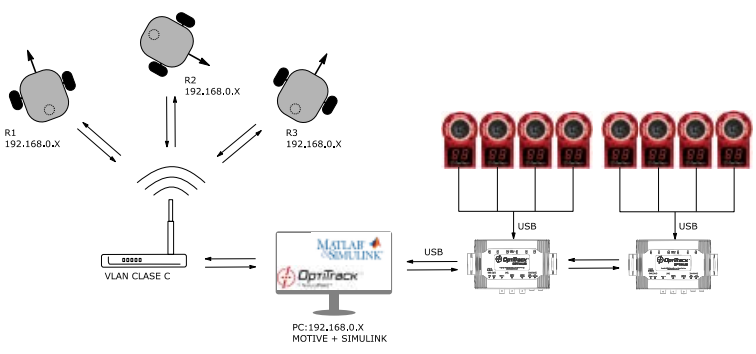

Figura 9: Comunicación entre los elementos del laboratorio

Las referencias de posicionamiento, calculadas por el algoritmo de control a partir de los datos obtenidos por las cámaras, se envían desde el $\mathrm{PC}$ a los robots en forma de coordenadas e indican el punto al que deben desplazarse los robots [11].

\subsection{RESULTADOS MEJORADOS}

Con el objetivo de ilustrar los errores derivados de la estimación de la posición mediante los encoders, se utilizó el sistema de visión presentado anteriormente para registrar las posiciones reales de los robots durante el primer experimento. Como se puede comprobar en la Figura 10, la trayectoria capturada por las cámaras difiere respecto a la estimada por los encoders. Estas diferencias se hacen patentes principalmente en los giros.

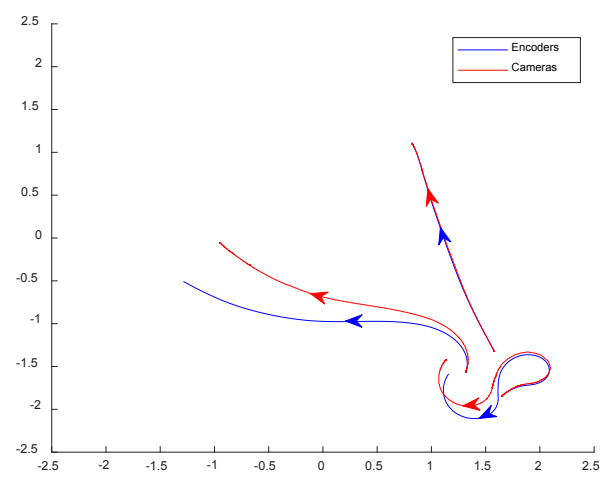

Figura 10: Comparación entre las trayectorias estimadas por los encoders y las trayectorias reales captadas por las cámaras

Para confirmar la efectividad del sistema de posicionamiento por visión, se realiza un segundo experimento empleando como realimentación las posiciones reales obtenidas con este sistema. Al finalizar el ensayo puede apreciarse una mayor uniformidad en el tamaño de las celdas (ver Figura 11), debido a que el algoritmo de cobertura trabaja con mayor precisión. 

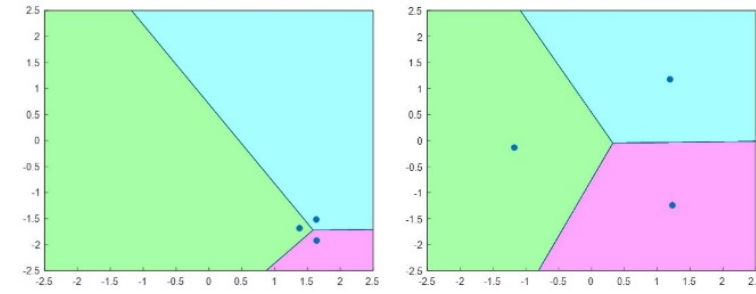

Figura 11: Distribución inicial y final de las celdas para cada robot cuando se emplea la información de las cámaras en la realimentación

Aunque las áreas de las celdas en la situación final de este segundo experimento están más compensadas que en el primero, el espacio no queda perfectamente repartido. La diferencia entre el tamaño final de las tres celdas es bastante notoria, ya que el área de trabajo es de solo $5 \mathrm{~m} \times 5 \mathrm{~m}$. Lo habitual para este tipo de sistema es que se utilicen áreas extensas, por lo que esta diferencia sería prácticamente inapreciable. Este problema se debe a que los robots mantienen una distancia de seguridad de $25 \mathrm{~cm}$ respecto al punto objetivo para asegurar un mejor comportamiento. Observando la Figura 5, se comprueba que ninguno de los robots termina su trayectoria sobre el final del trazo negro (puntos de referencia), lo cual supondría una reducción del tamaño de la celda asignada al robot situado en la parte izquierda, en beneficio de las otras dos zonas.

\section{CONCLUSIONES}

La posibilidad de trabajar de manera conjunta con Motive y Simulink, es decir, disponer de toda la información generada por el sistema en un único software proporciona numerosos beneficios en el entorno educativo, al no tener el alumno que enfrentarse a una nueva plataforma de desarrollo. Mediante el sistema de captura de datos por visión, pueden realizarse prácticas centradas en el modelado y control de robots móviles aéreos y terrestres, en el desarrollo de sistemas de localización y navegación, en el estudio de los problemas generados por la odometría en robots terrestres y en el control coordinado de flotas de robots móviles.

Los experimentos realizados muestran la importancia de disponer de un sistema que permita determinar con fidelidad la posición de los robots móviles, ya que, en caso contrario, los resultados de las pruebas se degradan con facilidad. Este problema es mayor cuando se efectúan ensayos en los que los robots pueden colisionar por la acumulación de errores en la estimación de sus posiciones.

\section{Agradecimientos}

Los autores agradecen la ayuda prestada por el Gobierno de La Rioja a través del proyecto de I+D ADER 2017-I-IDD-00035 y por la Universidad de La Rioja a través de la Ayuda a Grupos de Investigación REGI 2018/42.

\section{English summary}

\section{IMPROVEMENT OF COORDINATED CONTROL SYSTEM FOR MOBILE ROBOTS BY USING A VISION-BASED LOCALIZATION SYSTEM}

\begin{abstract}
The paper presents a coverage coordinated control system using Lloyd's algorithm in which three LEGO Mindstorms EV3 robots are involved, as a practical example of educational robotics. The application is developed in MATLAB-Simulink, a well-known environment usually used for control of dynamic systems. The purpose is to show the problems caused by the use of inaccurate localization systems, in this case, the encoders incorporated in the motors of the robots. A vision-based localization system solves this problem. The hardware and software infrastructure used in the laboratory that contains the vision-based system is explained. This whole set provides a useful low-cost platform for localization.
\end{abstract}

Keywords: Coordinated control, Coverage control, Mobile robots, Vision-based localization system.

\section{Referencias}

[1] Arai, T., Pagello, E., Parker, L. E.: Editorial: Advances in Multi-Robot Systems. IEEE Transactions on Robotics and Automation 18(5), 655-661 (2002).

[2] Atiya, S., Hager, G.: Real-Time Vision-Based Robot Localization. IEEE Transactions on Robotics and Automation 9(6), 785-800 (1993).

[3] Balch, T., Hybinette, M.: Social Potentials for Scalable Multi-Robot Formations. Proceedings of the IEEE International Conference on Robotics \& Automation, pp. 73-80. San Francisco, USA, April 2000. 
[4] Behrens, A., Atorf, L., Schwann, R., Neumann, B., Schnitzler, R., Ballé, J., Herold, T., Telle, A., Noll, T. G., Hameyer, K., Aach, T.: MATLAB Meets LEGO Mindstorms - A Freshman Introduction Course Into Practical Engineering. IEEE Transactions on Education 53(2), 306-317 (2010).

[5] Bullo, F., Cortés, J., Martínez, S.: Chapter 5: Deployment. In: Princeton University Press, Princeton and Oxford. Distributed Control of Robotic Networks, A Mathematical Approach to Motion Coordination Algorithms, pp. 5-43 (2019).

[6] Cañas, J. M., Martín, A., Perdices, E., Rivas, F., Calvo, R.: Entorno Docente Universitario para la Programación de Robots. Revista Iberoamericana de Automática e Informática Industrial 15, 404-415 (2018).

[7] Cortés, J., Egerstedt, M.: Coordinated Control of Multi-Robot Systems: A Survey. SICE Journal of Control, Measurement, and System Integration 10(6), 495-503 (2017).

[8] Dhaouadi, R., Hatab, A.A.: Dynamic Modelling of Differential-Drive Mobile Robots using Lagrange and Newton-Euler Methodologies: A Unified Framework. ICRA 2013 2(2), (2013).

[9] Du, Q., Faber, V., Gunzburger, M.: Centroidal Voronoi Tessellations: Applications and Algorithms. SIAM Review 41(4), 637-676 (1999).

[10] Elya, M. N., Mohd Noor S. B., Ribhan Zafira A. R., Azrad, S.: Implementation of Extended HighGain Observer in Low-Cost Optitrack Motion Tracking System for UAV control. IEEE 15th Student Conference on Research and Development, 29-33 (2017).

[11] Espinosa, F., Lázaro, J. L., Olivares, J.: Proyecto ALCOR: Contribuciones a la Optimización del Guiado Remoto de Robots en Espacios Inteligentes. Revista Iberoamericana de Automática e Informática Industrial 15, 416-426 (2018).

[12] Gallarta, D., Rico-Azagra, J., Nájera, S., GilMartínez, M.: Laboratorio para el desarrollo de sistemas de navegación y control para UAVs multirrotor. Actas del XXXIX Jornadas de Automática, pp. 613-620. Badajoz, España, Septiembre 2018.
[13] Galvan, S., Botturi, D., Castellani, A., Fiorini, P.: Innovative Robotics Teaching Using Lego Sets. Proceedings of the IEEE International Conference on Robotics and Automation, pp. 721-726. Orlando, USA, May 2006.

[14] González, J., Ollero, A.: Estimación de la Posición de un Robot Móvil. Informática y automática: revista de la Asociación Española de Informática y Automática 29(4), 3-18 (1996).

[15] Mastellone, S., Stipanović, D., Spong, M.: Remote Formation Control and Collision Avoidance for Multi-Agent Nonholonomic Systems. Proceedings of the IEEE International Conference on Robotics and Automation, pp. 1062-1067. Roma, Italy, April 2007.

[16] Pandey, A., Pandey, S., Parhi, D. R.: Mobile Robot Navigation and Obstacle Avoidance Techniques: A Review. International Journal of Robotics and Automation 2(3), (2017).

[17] Ranjbar-Sahraei, B., Weiss, G., Nakisaei, A.: A Multi-Robot Coverage Approach based on Stigmergic Communication. German Conference on Multiagent System Technologies 7589, 126-138 (2012).

[18] Rico-Azgra, J., Gil-Martínez, M., Rico-Azagra, R., Maisterra,P.: Low-cost attitude estimation for a ground vehicle. Robot 2015: Second Iberian Robotics Conference, Advances in Intelligent Systems and Computing, 121-132 (2016).

[19] Valera, A., Soriano, A., Vallés, M.: Plataformas de Bajo Coste para la Realización de Trabajos Prácticos de Mecatrónica y Robótica. Revista Iberoamericana de Automática e Informática Industrial 11, 363-376 (2014).

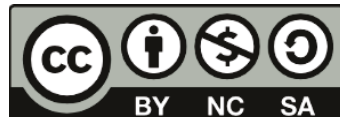

(C) 2019 by the authors. Submitted for possible open access publication under the terms and conditions of the Creative Commons Attribution CC BY-NC-SA 4.0 license (https://creativecommons.org/licenses/bync-sa/4.0/deed.es). 